\title{
Immunohistochemical profile of ING3 protein in normal and cancerous tissues
}

\author{
WEN-FENG GOU ${ }^{1}$, XUE-FENG YANG ${ }^{1}$, DAO-FU SHEN ${ }^{1}$, SHUANG ZHAO ${ }^{1}$, \\ HONG-ZHI SUN ${ }^{1}$, JUN-SHENG LUO ${ }^{2}$ and HUA-CHUAN ZHENG ${ }^{1,2}$ \\ ${ }^{1}$ Cancer Center and ${ }^{2}$ Key Laboratory of Brain and Spinal Cord Injury of Liaoning Province, \\ The First Affiliated Hospital of Liaoning Medical University, Jinzhou, Liaoning 121001, P.R. China
}

Received December 31, 2014; Accepted December 14, 2015

DOI: $10.3892 / \mathrm{ol} .2017 .5632$

\begin{abstract}
The inhibitor of growth family, member 3 (ING3) protein may be capable of blocking the cell cycle via activating p53-transactivated promoters of p21 and Bcl2-associated X protein, and may induce apoptosis via a Fas/caspase-8-dependent signaling pathway. In the present study, immunohistochemistry was performed in order to characterize the expression profile of ING3 protein in tissue microarrays containing mouse and human normal tissue, human hepatocellular $(n=62)$, renal clear cell $(n=62)$, pancreatic $(n=62)$, esophageal squamous cell $(n=45)$, cervical squamous cell $(n=31)$, breast $(n=144)$, gastric $(n=196)$, colorectal $(n=96)$, ovarian $(n=208)$, endometrial $(n=96)$ and lung carcinoma $(n=192)$. In mouse tissue, ING3 protein was positively detected in the cytoplasm of cardiomyocytes, kidney and skeletal muscle cells, and was additionally detected in the cytoplasm and nucleus of bronchial and alveolar epithelium, gastric and intestinal gland, and mammary gland cells. In human tissues, ING3 protein was principally distributed in the cytoplasm, but was observed in the cytoplasm and nucleus of tongue, esophagus, stomach, intestine, lung, skin, appendix, bladder, cervix and breast cells. ING3 immunoreactivity was strongly detected in the stomach, skin and cervical tissues, whereas a weak signal was detected in the cerebellum, brain stem, thymus, liver, skeletal muscle, testis and prostate. In total, ING3-positive specimens were identified in 424 of 1,194 tested cancer entities (35.5\%). In a number of cases, ING3 expression was observed to be restricted to the cytoplasm and nucleus, excluding the cytoplasmic distribution identified in breast and hepatocellular carcinoma. Among these cases, ING3 was more frequently expressed in breast and gynecological types of cancer, including ovarian (59.2\%), endometrial (47.9\%), breast (38.9\%) and cervical (35.5\%) cancer. ING3-positive cases were
\end{abstract}

Correspondence to: Professor Hua-Chuan Zheng, Cancer Center, The First Affiliated Hospital of Liaoning Medical University, 5-2 Renmin Street, Jinzhou, Liaoning 121001, P.R. China

E-mail: zheng_huachuan@hotmail.com

Key words: inhibitor of growth family member 3, expression profile, immunohistochemistry, mouse, human, cancer more rare in renal clear cell (17.7\%), hepatocellular (16.1\%) and esophageal carcinoma (17.8\%). It is suggested that ING3 may be involved in the repair and regeneration of organs or tissues, and may be closely associated with gynecological carcinogenesis.

\section{Introduction}

The inhibitor of growth (ING) family consists of five members with various isoforms due to alternative splicing. Their encoded proteins comprise a highly conserved plant homeodomain (PHD), a $\mathrm{Cys}_{4}-\mathrm{His}-\mathrm{Cys}_{3}$ form of zinc finger that interacts directly with histone $\mathrm{H} 3$, and a nuclear localization sequence (NLS). ING proteins act as receptors and transducers of stress-activated phosphoinositides, inhibit angiogenesis, promote cellular senescence or are involved in various biological processes, including DNA repair, apoptosis, cell cycle checkpoints, histone methylation and acetylation, and regulation of transcription by protein-protein or protein-DNA interaction. They appear to be inactivated in malignancies and therefore are classified as class II tumor suppressor genes $(1,2)$.

The human ING3 gene is located at chromosome 7q31.3, is composed of 12 exons and produces three proteins (Fig. 1), among which p47ING3 controls p53-mediated transcription, blocks cell cycle control and induces apoptosis (3). As a significant chromatin acetylation regulator, ING3 is primarily involved in the formation of nucleosome $\mathrm{NuA} 4$ histone acetyltransferase multi-subunit complex and is essential for the histone acetyltransferase activity of Tip60 $(2,4)$. ING3 overexpression decreases the S-phase population of cells and their colony-forming ability, and induces apoptosis in RKO human colon carcinoma cells in a p53-mediated manner (5). An additional study has indicated that ING3 activates p53-transactivated promoters of p21 and Bcl2-associated $X$ protein (5). In addition, ING3 has been demonstrated to be capable of enhancing ultraviolet-induced apoptosis of melanoma cells via a Fas/caspase-8-dependent signaling pathway, independently of functional p53 (6). Furthermore, it was identified that ING3 underwent degradation via its interaction with subunits of E3 ligase Skp1-Cullin-F-box (SCF) protein complex in the ubiquitin-proteasome signaling pathway, which provided an alternative explanation for ING3 downregulation (7). ING3 may also be capable of regulating asymmetric cell division via the mammalian target of rapamycin signaling pathway during mouse oocyte maturation (8). 


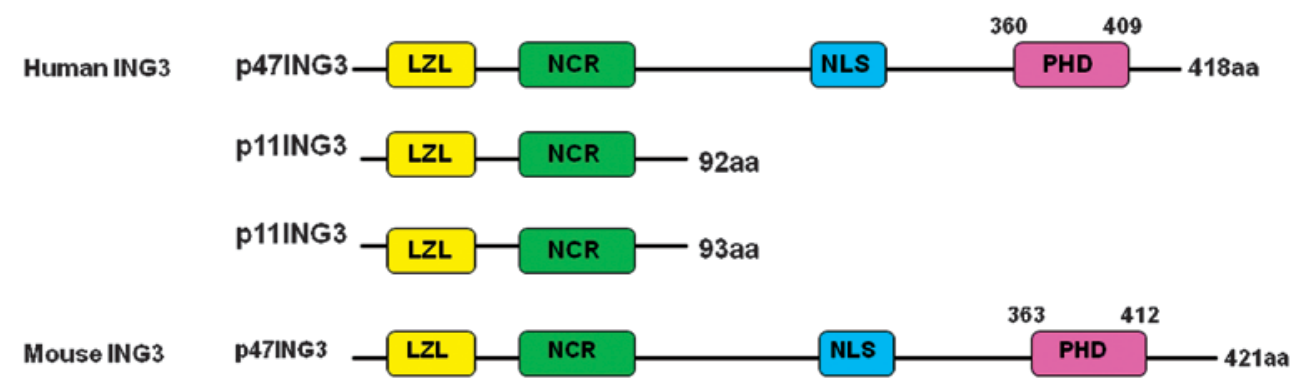

Figure 1. Schematic diagram of human and mouse inhibitor of growth family, member 3 proteins. LZL, leucine zipper-like; NCR, novel conserved region; NLS, nuclear localization signal, PHD, plant homeodomain.

A

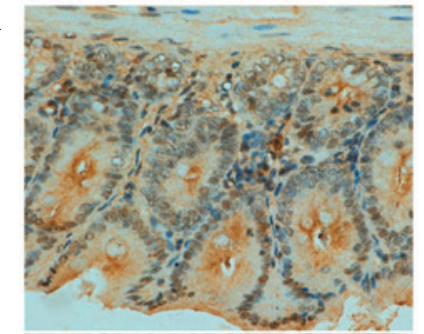

C

$\mathbf{E}$

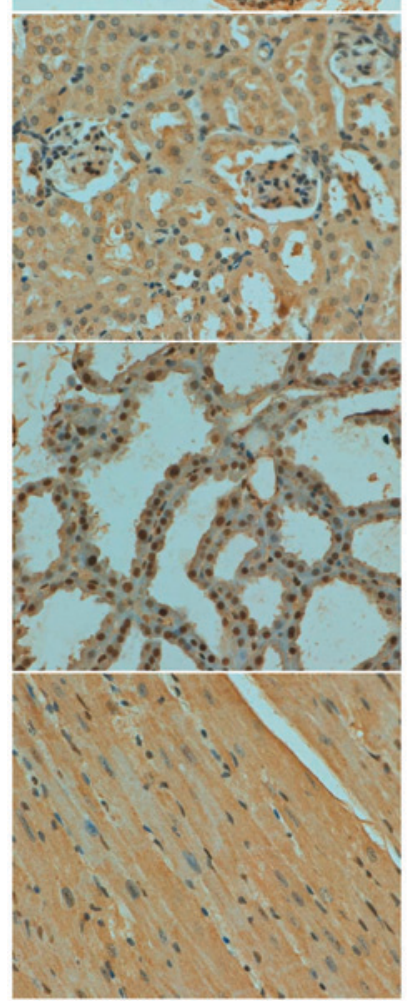

B

D
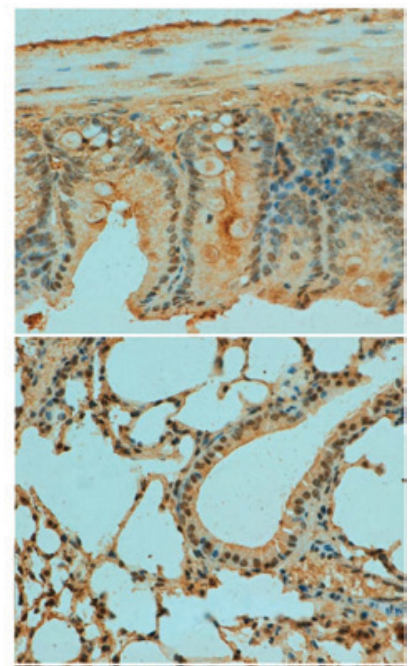

$\mathbf{F}$

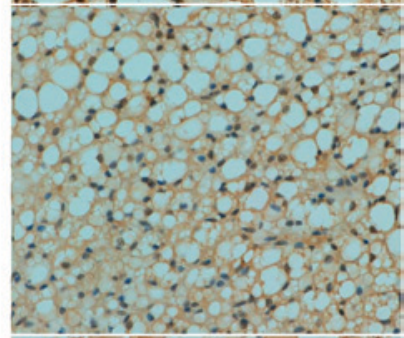

$\mathbf{H}$

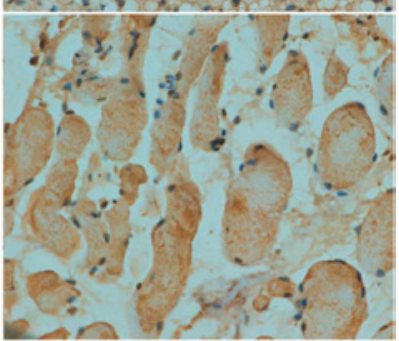

Figure 2. Expression level and subcellular distribution of inhibitor of growth family, member 3 protein in normal mouse tissues: (A) Stomach, (B) intestine, (C) kidney, (D) lung and trachea, (E) breast, (F) fat, (G) cardiac muscle and (H) striated muscle. Magnification, x200.

ING3 mRNA is ubiquitously expressed in normal human tissues, including the testes, skeletal muscle, spleen, heart and oral mucosa (5). Nuclear ING3 expression is markedly reduced in malignant melanoma compared with dysplastic nevi, and is significantly associated with a poorer prognosis for melanoma as an independent factor (9). Gunduz et al (10) demonstrated that loss of heterozygosity ( $\mathrm{LOH}$ ) resulted in reduced ING3 expression in human head and neck squamous cell carcinomas (HNSCC). A previous survival analysis revealed that ING3 downregulation may be considered as an independent prognostic factor for poor overall survival time in HNSCC (11). In addition, Borkosky et al (12) identified that SSLOH of the ING3 locus was high in solid type tumors of ameloblastoma. mRNA and protein concentrations of ING3 have been observed to be downregulated in the majority of hepatocellular carcinoma (HCC) cases in comparison with matched non-tumor hepatic tissues, and reduced expression of ING3 protein is correlated with more aggressive characteristics and adverse prognosis in this tumor type $(13,14)$. Consistently, ectopic ING3 overexpression in HCC cells was 
Table I. Immunohistochemical examination of inhibitor of growth family, member 3 protein in mouse normal tissues.

\begin{tabular}{ll}
\hline Tissue type & \multicolumn{1}{c}{ Cell type } \\
\hline Brain & Sporadic \\
Heart & Cardiomyocyte \\
Lung & Bronchial and alveolar epithelium \\
Kidney & Nephric tubule \\
Stomach & Glandular \\
Intestine & Glandular \\
Spleen & Sporadic \\
Skin & Sporadic \\
Muscle & Striated muscle cell \\
Fat & Lipocyte \\
Liver & Sporadic \\
Breast & Glandular epithelium
\end{tabular}

Sporadic refers to the expression of inhibitor of growth family, member 3 protein in different cell types.

observed to suppress colony formation, cell proliferation and migration $(13,14)$. These results suggest that reduced ING3 expression may be associated with tumorigenesis and the subsequent development of malignancies. Thus, the present study analyzed the expression profile of ING3 protein in normal mouse and human tissues, and in human cancer tissues.

\section{Materials and methods}

Samples. A total of three male and three female C57BL/6 mice ( 8 weeks old; 30-40 g) were maintained under specific pathogen-free conditions in a temperaturecontrolled room with a 12 -h light/dark illumination cycle. Standard rodent food and water were supplied ad libitum. Housing and all procedures were performed according to guidelines on animal welfare approved by the Committee for Animal Experiments of Liaoning Medical University. The mice were sacrificed under sodium pentobarbital anesthesia, and tissue samples were dissected from the brain, heart, liver, spleen, lung, kidney, breast, stomach and intestine. All tissues were fixed in $10 \%$ neutral formalin, embedded in paraffin and cut into $4-\mu \mathrm{m}$ sections. The tissue arrays of human normal tissues (cerebrum, cerebellum, brain stem, aorta, tongue, thyroid, esophagus, stomach, intestine, liver, pancreas, lung, trachea, appendix, smooth muscle, skeletal muscle, heart, testis, bladder and prostate) and cancer tissues (62 hepatocellular carcinoma, 62 renal clear cell carcinoma, 62 pancreatic carcinoma, 45 esophageal squamous cell carcinoma and 31 cervical squamous cell carcinoma cases) were purchased from Shanghai Outdo Biotech Co., Ltd (Shanghai, China). Human cervix, endometrium, ovary and breast tissues were sampled from surgical patients at The First Affiliated Hospital of Liaoning Medical University (Jinzhou, China). In addition, breast $(n=144)$, gastric $(n=196)$, colorectal $(n=96)$, ovarian $(n=208)$, endometrial $(n=96)$
Table II. Immunostaining of ING3 protein in normal human tissues.

\begin{tabular}{|c|c|c|}
\hline \multirow[b]{2}{*}{ Tissue type } & \multicolumn{2}{|c|}{ ING3 expression } \\
\hline & Nucleus & Cytoplasm \\
\hline Cerebrum & - & + \\
\hline Cerebellum & - & + \\
\hline Brain stem & - & + \\
\hline Thymus & - & + \\
\hline Hear muscle & - & + \\
\hline Aorta & - & + \\
\hline Tongue & + & + \\
\hline Thyroid & - & + \\
\hline Esophagus & + & + \\
\hline Stomach & + & + \\
\hline Intestine & + & + \\
\hline Liver & - & + \\
\hline Pancreas & - & + \\
\hline Lung & + & + \\
\hline Trachea & - & + \\
\hline Skin & + & + \\
\hline Appendix & + & + \\
\hline Smooth muscle & - & + \\
\hline Skeletal muscle & - & + \\
\hline Heart & - & + \\
\hline Testis & - & + \\
\hline Bladder & + & + \\
\hline Prostate & - & + \\
\hline Cervix & + & + \\
\hline Endometrium & + & + \\
\hline Ovary & - & + \\
\hline Breast & + & + \\
\hline
\end{tabular}

ING3, inhibitor of growth family, member 3 .

and lung carcinoma $(n=192)$ samples were collected from patients at the same hospital. Dissected mouse tissues and collected human normal and cancer tissues, were subjected to tissue microarray using a tissue microarrayer (AZUMAYA KIN-1; Azumaya Corporation, Tokyo, Japan). None of the cancer patients had undergone chemotherapy, radiotherapy or adjuvant treatment prior to surgery. The patients or their relatives provided written consent for the use of tumor tissues for clinical research, and the research protocol was approved by the Ethical and Animal Experimentation Committees of Liaoning Medical University (Jinzhou, China).

Immunohistochemistry. Consecutive sections were dewaxed using xylene, rehydrated in a graded series of alcohol to water, and subjected to antigen retrieval by irradiation in target retrieval solution (Dako North America, Inc., Carpinteria, CA, USA) in a microwave oven for $15 \mathrm{~min}$ (Oriental Rotor Ltd., Co., Tokyo, Japan). Sections were subsequently blocked with 5\% bovine serum albumin (A8020; Beijing Solarbio 
Table III. ING3 expression in various types of cancer detected by immunohistochemistry.

ING3 expression

\begin{tabular}{lccccc}
\cline { 3 - 5 } Carcinoma type & Total cases, $\mathrm{n}$ & Positive cases, $\mathrm{n}$ & Positive rate, $\%$ & Nucleus & Cytoplasm \\
\hline Hepatocellular & 62 & 11 & 17.7 & - & + \\
Renal clear cell & 62 & 10 & 16.1 & + & + \\
Pancreatic & 62 & 23 & 37.1 & + & + \\
Esophageal & 45 & 8 & 17.8 & + & + \\
Cervical & 31 & 56 & 35.5 & - & + \\
Breast & 144 & 57 & 38.9 & + & + \\
Gastric & 196 & 28 & 29.1 & + & + \\
Colorectal & 96 & 124 & 29.2 & + & + \\
Ovarian & 208 & 46 & 59.6 & + & + \\
Endometrial & 96 & 50 & 47.9 & + & + \\
Lung & 192 & 26.0 & + \\
\hline
\end{tabular}

ING3, inhibitor of growth family, member 3.

Science \& Technology Co., Ltd., Beijing, China) for $20 \mathrm{~min}$ to prevent non-specific antibody binding. The sections were incubated with rabbit polyclonal IgG anti-ING3 (\#sc-366026; Santa Cruz Biotechnology, Inc., Dallas, TX, USA; dilution, 1:50) for $15 \mathrm{~min}$, followed by incubation with the secondary anti-rabbit polyclonal $\mathrm{Ig}$ antibody conjugated to horseradish peroxidase (HRP) (\#P0399; HRP; Dako North America, Inc.; ready-to-use) for $60 \mathrm{~min}$. Following each treatment, the slides were washed using Tris-buffered saline and Tween 20 (TBST; 3 x 1-min washes). The HRP was colored with 3,3'-diaminobenzidine. Sections were counterstained using Mayer's hematoxylin, dehydrated, cleared and mounted. TBST was utilized as a negative control in place of primary antibody.

Immunohistochemical evaluation. As indicated in Figs. 2-4, ING3 immunopositivity was localized to the cytoplasm and/or nucleus. Initially, a strong expression field was selected under low magnification and all cells were randomly counted in five different representative fields of each section, which were assessed blindly by two independent pathologists. Any inconsistent data was discussed by the pathologists until a final agreement was reached. The percentages of counted cells (calculated as the mean percent of positively stained cells out of the total cells counted) were scored as follows: $0-10 \%$, negative $(-), 11-100 \%$, positive $(+)$.

\section{Results}

ING3 is detectable in a wide range of cell types in mice. As indicated in Fig. 2, ING3 protein was positively detected in the cytoplasm of cardiomyocyte, kidney and skeletal muscle cells. A cytoplasmic and nuclear distribution of ING3 protein was observed in bronchial and alveolar epithelium, gastric, intestinal and mammary gland cells. ING3 protein was expressed in the brain, spleen, skin and liver in a sporadic manner (Table I).

ING3 expression is primarily localized to the cytoplasm in normal human tissues. In human tissues, ING3 protein was principally distributed in the cytoplasm; however, it was observed in both the cytoplasm and nucleus of tongue, esophagus, stomach, intestine, lung, skin, appendix, bladder, cervix and breast cells (Table II; Fig. 3). According to the density, ING3 immunoreactivity was strongly detected in stomach, skin and cervical cells, and was weakly detected in cerebellum, brain stem, thymus, liver, skeletal muscle, testis and prostate cells (data not shown).

ING3 is most frequently expressed in gynecological types of cancer. In total, ING3-positivity was identified in 424 of 1,194 tested cancer entities (35.5\%), with a homogeneous expression pattern (Fig. 4; Table III). In the majority of cases, ING3 expression was observed to be distributed in the cytoplasm and nucleus, with the exception of the cytoplasmic distribution in breast and hepatocellular carcinoma. Among the cancer entities studied, ING3 was most frequently expressed in cases involving female cancer types, including ovarian $(59.2 \% ; 124 / 208)$, endometrial $(47.9 \%$; $46 / 96)$, breast $(38.9 \%$; 56/144) and cervical cancers $(35.5 \%$; 11/31). ING3-positive cases were more rare in renal clear cell $(17.7 \% ; 11 / 62)$, hepatocellular $(16.1 \% ; 10 / 62)$ and esophageal carcinomas $(17.8 \% ; 8 / 45)$.

\section{Discussion}

ING3 protein contains an NLS and a PHD finger motif at the C-terminus (15). Previously, Wang et al (9) demonstrated that nuclear-to-cytoplasmic translocation of ING3 protein led to reduced nuclear expression in cutaneous melanoma. The degradation of ING3 by the cytoplasmic SCF (S-phase kinase-associated protein 2)-mediated ubiquitin-proteasome system provided additional evidence for its cytosolic localization (7). An additional two studies observed a cytoplasmic expression pattern of ING3 in hepatocytes and HCC $(13,14)$. In the present study, the expression level and cellular localization of ING3 protein was characterized in normal mouse and human tissue, and human cancer tissue. A positive ING3 signal was 

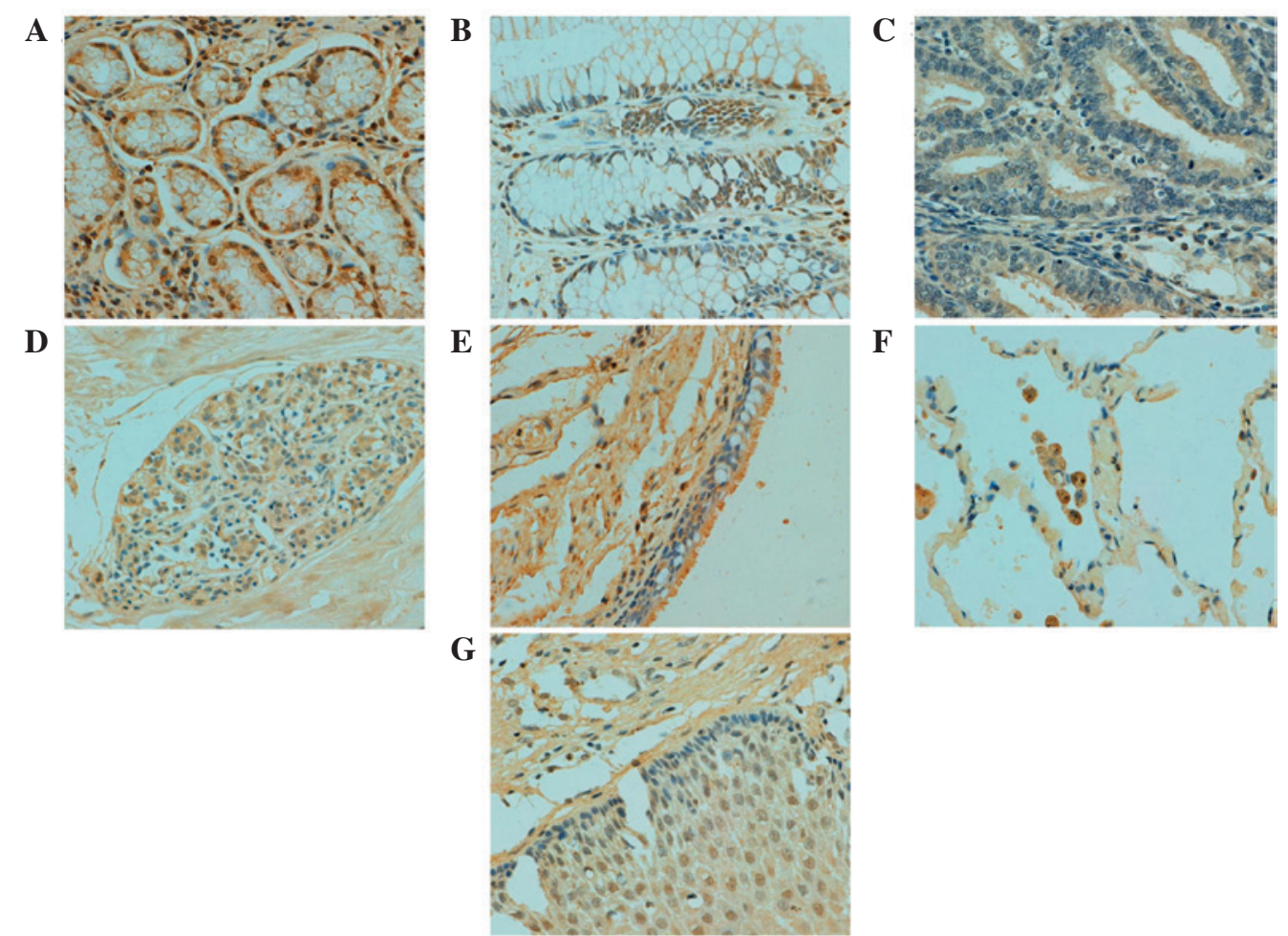

Figure 3. Expression level and subcellular distribution of inhibitor of growth family, member 3 protein in normal human tissues: (A) Stomach, (B) intestine, (C) endometrium, (D) breast, (E) bronchus, (F) lung and (G) cervix. Magnification, x200.

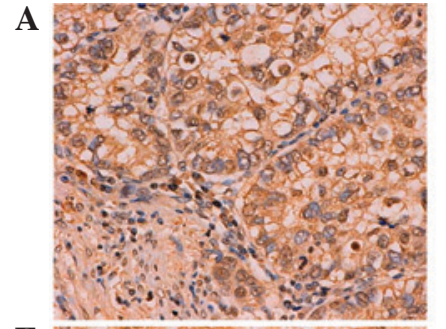

E

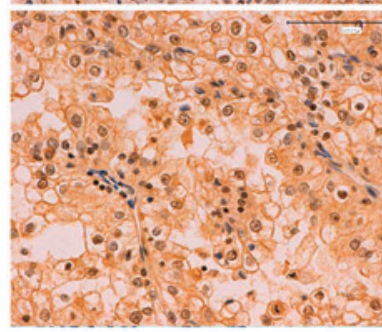

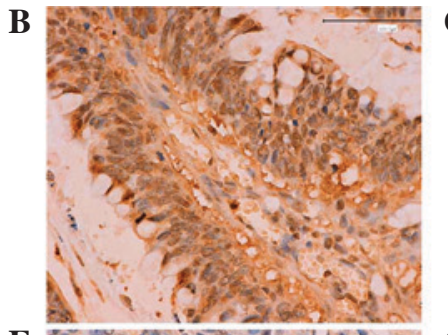

F

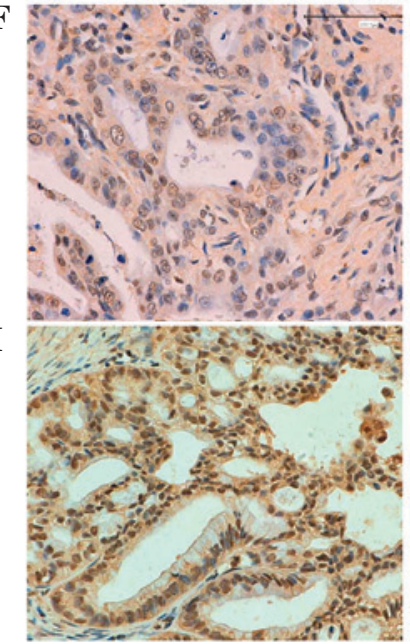

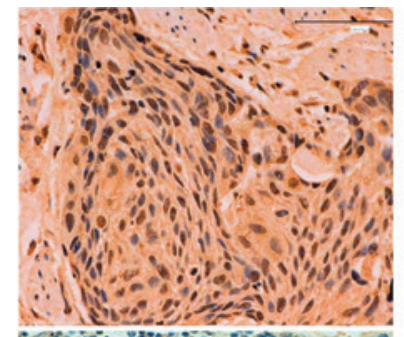

$\mathbf{G}$

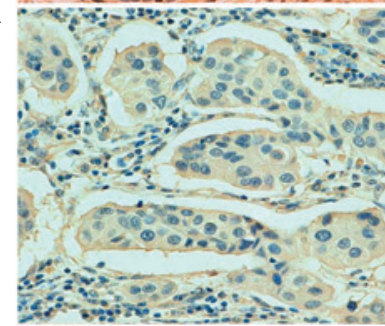

\section{$\mathbf{J}$}

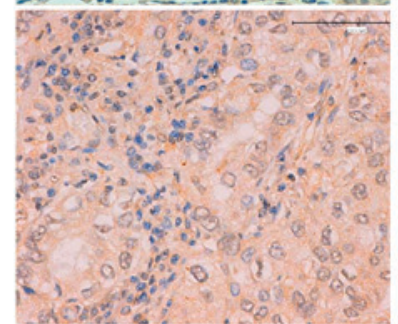

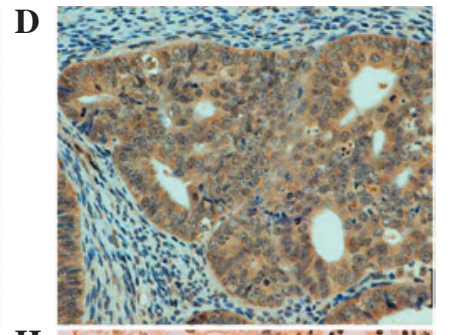

$\mathbf{H}$

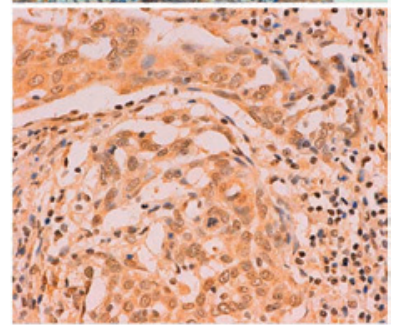

Figure 4. Inhibitor of growth family, member 3 expression in human cancer detected by immunohistochemistry: (A) Gastric, (B) colorectal, (C) esophageal, (D) endometrial, (E) renal, (F) pancreatic, (G) breast, (H) cervical, (I) ovarian and (J) lung carcinoma. Magnification, x200.

observed in the cytoplasm of normal mouse and human tissue, and in human cancer tissue, and was occasionally observed in both the cytoplasm and nucleus. Cenzig et al (16) reported that the mutation or deletion of the ING5 NLS resulted in its nucleocytoplasmic translocation. ING1 phosphorylation by 14-3-3 family (17) or Src (18) proteins leads to its cytoplasmic relocalization for apoptotic induction. Therefore, it was speculated that chemical modification of ING3 may lead to its 
restoration in the cytoplasm, which will require clarification in future studies.

Amino acid sequence alignment has demonstrated a high similarity between human p47ING3 and mouse ING3, revealing that they share $95 \%$ identity (1). Consistently, the present study identified no notable differences in the patterns of ING3 expression between mouse and human samples. In human tissue, ING3 protein was strongly detected in stomach, skin and cervical cells, and was weakly detected in brain, thymus, liver, skeletal muscle, testis and prostate cells, suggesting a functional involvement of ING3 in distinct cell types and in the specific functional state of cells. Therefore, in future studies, we aim to conditionally ablate the ING3 gene using a cell-specific promoter and establish an animal model of ING3-negative tumors. In the relevant literature, ectopic ING3 expression resulted in increased apoptosis via the Fas-mediated signaling pathway (6) and suppression of proliferation (5). Therefore, ING3 overexpression in the stomach, skin and cervix may be associated with regeneration and repair, regardless of whether glandular or squamous epithelium; this is supported by the observed weaker expression in organs with low levels of repair and renewal, including the brain, thymus, skeletal muscle and testis. Notably, ING2, another member of the ING family, has been reported to be involved in muscle differentiation via regulating myogenin transcription (19). As a member of the ING family, ING3 protein is enriched in heart, skeletal and smooth muscle cells, which is hypothesized to be associated with the differentiation of muscles.

ING3 is a candidate tumor suppressor gene, and its expression is frequently downregulated in tumors $(9,14,15)$. The present study focused on the most commonly occurring epithelial cancers and demonstrated that female types of cancer, including breast, ovarian and endometrial, exhibited higher levels of ING3 expression, indicating that ING3 protein may be involved in estrogen production or may be regulated by estrogen. It was notable that gastric and colorectal cancers demonstrated similar levels and patterns of expression of ING3, which may be due to the similar carcinogenesis and pathological behaviors of these types of cancer. By contrast, renal clear cell carcinoma demonstrated the lowest levels of ING3 expression, with a positive rate of $<20 \%$. This knowledge may significantly facilitate the identification of cancer patients that may potentially benefit from an ING3-targeting gene therapy. According to the relevant literature, ING3 protein is involved in the modulation of p53-mediated transcription, cell cycle control and apoptosis $(1,2)$. In RKO human colon carcinoma cells, ING3 overexpression reduced colony formation, potentially by reducing the number of cells in $\mathrm{S}$ phase (5). In combination with these findings, the profiling of ING3 expression may assist with clarification of the role of ING3 expression in disruption of proliferation and apoptosis in various types of epithelial cancer.

In summary, the present study clarified the differential expression and/or subcellular location of ING3 in various tissues, cell types and single cells in normal mouse and human tissues, and human cancer tissue, suggesting differential functional involvement. Based on the results of the present study, it is hypothesized that ING3 may be involved in the repair and regeneration of organs or tissues, and may have a significant role in gynecological carcinogenesis.

\section{Acknowledgements}

This study was supported by the Liaoning BaiQianWan Talents Program, a Key Scientific and Technological Project of Liaoning Province (grant no. 2015408001), Scientific Research Fund of Liaoning Provincial Education Department (grant no. LJQ2014093) and the National Natural Science Foundation of China (grant nos. 81172371, 81472544 and 81672700).

\section{References}

1. Ludwig S, Klitzsch A and Baniahmad A: The ING tumor suppressors in cellular senescence and chromatin. Cell Biosci 1: 25, 2011.

2. Doyon Y, Cayrou C, Ullah M, Landry AJ, Côté V, Selleck W, Lane WS, Tan S, Yang XJ and Côté J: ING tumor suppressor proteins are critical regulators of chromatin acetylation required for genome expression and perpetuation. Mol Cell 21: 51-64, 2006.

3. Guérillon C, Bigot N and Pedeux R: The ING tumor suppressor genes: Status in human tumors. Cancer Lett 345: 1-16, 2014.

4. Ullah M, Pelletier N, Xiao L, Zhao SP, Wang K, Degerny C, Tahmasebi S, Cayrou C, Doyon Y, Goh SL, et al: Molecular architecture of quartet MOZ/MORF histone acetyltransferase complexes. Mol Cell Biol 28: 6828-6843, 2008.

5. Nagashima M, Shiseki M, Pedeux RM, et al: A novel PHD-finger motif protein, p47ING3, modulates p53-mediated transcription, cell cycle control, and apoptosis. Oncogene 22: 343-350, 2003.

6. Wang Y and Li G: ING3 promotes UV-induced apoptosis via Fas/caspase-8 pathway in melanoma cells. J Biol Chem 281: 11887-11893, 2006.

7. Chen G, Wang Y, Garate M, Zhou J and Li G: The tumor suppressor ING3 is degraded by $\mathrm{SCF}(\mathrm{Skp} 2)$-mediated ubiquitin-proteasome system. Oncogene 29: 1498-1508, 2010.

8. Suzuki S, Nozawa Y, Tsukamoto S, Kaneko T, Imai H and Minami N: ING3 is essential for asymmetric cell division during mouse oocyte maturation. PLoS One 8: e74749, 2013.

9. Wang Y, Dai DL, Martinka M and Li G: Prognostic significance of nuclear ING3 expression in human cutaneous melanoma. Clin Cancer Res 13: 4111-4116, 2007.

10. Gunduz M,Ouchida M, Fukushima K, Ito S, Jitsumori Y, Nakashima T, Nagai N, Nishizaki K and Shimizu K: Allelic loss and reduced expression of the ING3, a candidate tumor suppressor gene at 7q31, in human head and neck cancers. Oncogene 21:4462-4470, 2002.

11. Gunduz M, Beder LB, Gunduz E, Nagatsuka H, Fukushima K, Pehlivan D, Cetin E, Yamanaka N, Nishizaki K, Shimizu K and Nagai N: Downregulation of ING3 mRNA expression predicts poor prognosis in head and neck cancer. Cancer Sci 99: 531-538, 2008.

12. Borkosky SS, Gunduz M, Beder L, Tsujigiwa H, Tamamura R, Gunduz E, Katase N, Rodriguez AP, Sasaki A, Nagai N and Nagatsuka H: Allelic loss of the ING gene family loci is a frequent event in ameloblastoma. Oncol Res 18: 509-518, 2010.

13. Lu M, Chen F, Wang Q, Wang K, Pan Q and Zhang X: Downregulation of inhibitor of growth 3 is correlated with tumorigenesis and progression of hepatocellular carcinoma. Oncol Lett 4: 47-52, 2012.

14. Yang HY, Liu HL, Tian LT, Song RP, Song X, Yin DL, Liang YJ, Qu LD, Jiang HC, Liu JR and Liu LX: Expression and prognostic value of ING3 in human primary hepatocellular carcinoma. Exp Biol Med (Maywood) 237: 352-361, 2012.

15. Shah S, Smith H, Feng X, Rancourt DE and Riabowol K: ING function in apoptosis in diverse model systems. Biochem Cell Biol 87: 117-125, 2009.

16. Cengiz B, Gunduz E, Gunduz M, Beder LB, Tamamura R, Bagci C, Yamanaka N, Shimizu K and Nagatsuka H: Tumor-specific mutation and downregulation of ING5 detected in oral squamous cell carcinoma. Int J Cancer 127: 2088-2094, 2010.

17. Gong W, Russell M, Suzuki K and Riabowol K: Subcellular targeting of p33ING1b by phosphorylation-dependent 14-3-3 binding regulates p21WAF1 expression. Mol Cell Biol 26: 2947-2954, 2006.

18. Yu L, Thakur S, Leong-Quong RY, Suzuki K, Pang A, Bjorge JD, Riabowol K and Fujita DJ: Src regulates the activity of the ING1 tumor suppressor. PLoS One 8: e60943, 2013.

19. Eapen SA, Netherton SJ, Sarker KP, Deng L, Chan A, Riabowol K and Bonni S: Identification of a novel function for the chromatin remodeling protein ING2 in muscle differentiation. PLoS One 7: e40684, 2012 